\title{
An Assessment to Toxicological Risk of Pesticide Exposure
}

\author{
Cristina Coelho ${ }^{1}$, M. Rosário Martins ${ }^{2}$, Nelson Lima ${ }^{3}$, \\ Henrique Vicente ${ }^{1,4}$, and José Neves ${ }^{4(\bowtie)}$ \\ 1 Departamento de Química, Escola de Ciências e Tecnologia, \\ Universidade de Évora, Évora, Portugal \\ cristina.argente@gmail.com, hvicente@uevora.pt \\ 2 Departamento de Química, Laboratório HERCULES, \\ Escola de Ciências e Tecnologia, Universidade de Évora, Évora, Portugal \\ mrm@uevora.pt \\ ${ }^{3}$ Centro de Engenharia Biológica, Micoteca da Universidade do Minho, \\ Universidade do Minho, Braga, Portugal \\ nelson@ie.uminho.pt \\ ${ }^{4}$ Centro Algoritmi, Universidade do Minho, Braga, Portugal \\ jneves@di.uminho.pt
}

\begin{abstract}
On the one hand, pesticides may be absorbed into the body orally, dermally, ocularly and by inhalation and the human exposure may be dietary, recreational and/or occupational where toxicity could be acute or chronic. On the other hand, the environmental fate and toxicity of the pesticide is contingent on the physico-chemical characteristics of pesticide, the soil composition and adsorption. Human toxicity is also dependent on the exposure time and individual's susceptibility. Therefore, this work will focus on the development of an Artificial Intelligence based diagnosis support system to assess the pesticide toxicological risk to humanoid, built under a formal framework based on Logic Programming to knowledge representation and reasoning, complemented with an approach to computing grounded on Artificial Neural Networks. The proposed solution is unique in itself, once it caters for the explicit treatment of incomplete, unknown, or even self-contradictory information, either in terms of a qualitative or quantitative setting.
\end{abstract}

Keywords: Pesticide exposure $\cdot$ Toxicity $\cdot$ Environmental fate $\cdot$ Artificial intelligence $\cdot$ Logic programming $\cdot$ Knowledge representation and reasoning Artificial neuronal networks - Incomplete information 